\title{
SMART: An Efficient, Scalable, and Robust Streaming Video System
}

\author{
Feng Wu \\ Microsoft Research Asia, 3F Sigma Center, No. 49 Zhichun Road, Haidian, Beijing 100080, China \\ Email:fengwu@microsoft.com
}

\section{Honghui Sun}

Microsoft Research Asia, 3F Sigma Center, No. 49 Zhichun Road, Haidian, Beijing 100080, China Email: hongsun@microsoft.com

\section{Guobin Shen}

Microsoft Research Asia, 3F Sigma Center, No. 49 Zhichun Road, Haidian, Beijing 100080, China Email: jackysh@microsoft.com

\section{Shipeng Li}

Microsoft Research Asia, 3F Sigma Center, No. 49 Zhichun Road, Haidian, Beijing 100080, China Email: spli@microsoft.com

\section{Ya-Qin Zhang}

Microsoft Research Asia, 3F Sigma Center, No. 49 Zhichun Road, Haidian, Beijing 100080, China Email:yzhang@microsoft.com

\section{Bruce Lin}

Microsoft Corporation, One Microsoft Way, Redmond, WA 98052-6399, USA

Email: blin@microsoft.com

\section{Ming-Chieh Lee}

Microsoft Corporation, One Microsoft Way, Redmond, WA 98052-6399, USA

Email:mingcl@microsoft.com

Received 9 December 2002; Revised 12 September 2003

SMART, the acronym of scalable media adaptation and robust transport, is a suite of compression and transmission technologies for efficient, scalable, adaptive, and robust video streaming over the best-effort Internet. It consists of two indispensable parts: SMART video coding and SMART video streaming. The SMART video coding part is an efficient DCT-based universal fine granularity scalable coding scheme. Since the SMART video coding scheme adopts multiple-loop prediction and drifting reduction techniques at the macroblock level, it can achieve high coding efficiency at a wide range of bit rates. More importantly, it provides all sorts of scalabilities, that is, quality, temporal, spatial, and complexity scalabilities, in order to accommodate heterogeneous time-variant networks and different devices. The SMART video streaming part is a transport scheme that fully takes advantages of the special features of the scalable bitstreams. An accurate bandwidth estimation method is first discussed as the prerequisite of network adaptation. Then, flexible error resilience technique and unequal error protection strategy are investigated to enhance the robustness of streaming SMART bitstream. The SMART system shows excellent performances with regard to high coding efficiency, flexible channel bandwidth adaptation, smooth playback, and superior error robustness in static and dynamic experiments.

Keywords and phrases: video streaming, fine granularity scalability, video transmission, bandwidth estimation, error resilience, unequal error protection. 


\section{INTRODUCTION}

With the recent developments in computing technology, compression and transmission technologies, high-capacity storage devices, and high-speed wired and wireless networks, more and more users expect to enjoy high-quality multimedia services over the Internet $[1,2,3]$. In general, there are two approaches to provide multimedia services on demand: offline downloading and online streaming. Since the streaming approach enables users to experience a multimedia presentation on the fly while it is being downloaded from the Internet, it has prevailed in both the academia and the industry. In virtue of the streaming techniques, users no longer have to suffer from long and even unacceptable transport time for full download.

Figure 1 exemplifies a typical scenario for streaming the same content to users. Raw video sequences are usually compressed in advance and then saved in the storage device. Upon the client's request, the streaming server retrieves compressed bitstream from the storage device and delivers it through the Internet that consists of many heterogeneous subnetworks. Receivers may use different devices for decoding, presenting the received video data at different resolutions, different frame rates, and different qualities depending on their connection speeds and device capabilities.

In fact, such multimedia streaming services create several challenges which may lie in technical fields even beyond video compression. These challenges mainly include but are not limited to the following.

\section{(1) Contents}

Multimedia contents are huge and growing rapidly. For example, only from RealNetworks Company's statistics in 2001 [4], over 350000 hours of live sports, music, news, and entertainment contents were transmitted over the Internet every week. Furthermore, there are several hundred thousand hours of contents available on demand. To efficiently and effectively deliver such huge multimedia contents, advanced compression and transmission technologies are crucial.

\section{(2) Networks}

The networks used to deliver multimedia contents are becoming more and more complicated and heterogeneous. Additionally, unlike traditional dedicated networks, since the general best-effort Internet lacks quality of service (QOS) guarantee, network conditions themselves may be changing from time to time. This requires that compressed multimedia contents are deliverable over different networks from narrowband to broadband and from wired to wireless networks. It also requires that the delivery mechanism is able to adapt to network variations while providing a consistent user experience. In addition, since packet loss or channel error is inevitable during transmission, advanced error control technologies are required to protect the transmitted data.

\section{(3) Devices}

End-user devices are also becoming very different in processing power, memory, display resolution, and bandwidth. This requires tailoring multimedia contents and delivery schemes to best fit each device in order to provide the best possible multimedia user experience.

A straightforward solution would be to independently compress the same video sequence into many nonscalable bitstreams for every possible bit rate, frame rate, resolution, and device complexity. Actually, this solution has been extensively applied to most of the commercial streaming products, such as Windows Media Player system and Real Player system $[4,5]$. When a video sequence is retrieved, the streaming server chooses an appropriate version of bitstream according to actual connection speed and device capability, and then transmits it to the user.

Obviously, video streaming systems based on the nonscalable compression techniques have several problems in taking the above challenges. Firstly, nonscalable video bitstreams are not able to adapt to time-variant networks. Even though switching among multiple nonscalable bitstreams is allowed at some key frames that are either compressed without prediction or coded with an extra lossless coded switching bitstream, such streaming systems only provide coarse and sluggish capability in adapting to bandwidth variations due to limitation in both the number of bitstreams and the number of key frames. Some studies have tried to solve this problem by switching at a special predictive frame, for example, S frame in [6], SP frame in [7], and SF frame in [8], which can reduce switching overhead and provide more switching points at the same cost. Secondly, nonscalable video bitstream is very sensitive to transmitted errors because almost every bit in the bitstream is very important and indispensable for decoding a group of pictures (GOP).

On the other hand, the scalable media adaptation and robust transport (SMART) system proposed in this paper is based on scalable compression techniques and is able to provide efficient, adaptive, and robust video streaming over the Internet. The core of the system is an efficient and universal fine granularity scalable (FGS) video codec. It uses multiple versions of references with increasing quality to make motion prediction more accurate for improved coding efficiency. At the same time, a drifting reduction technique is proposed to prevent possible error propagation due to corrupted highquality references. When the two techniques are applied at the macroblock level, the SMART system can achieve a good trade-off between low drifting errors and high coding efficiency. Besides efficient fine granularity quality scalability, the SMART system supports efficient temporal and spatial scalabilities by utilizing similar techniques. Furthermore, the fine granularity scalability on complexity is also achieved by adjusting the decoding resolution, frame rate, and bit rate. In fact, the SMART system provides a universal scalable coding framework. For a sequence, the generated bitstreams can be served to a vast range of applications from low bit rate to high bit rate and from a PC device to a non-PC device without complicated transcoding.

The SMART video streaming part is a transport scheme that fully takes advantage of the special features of SMART video bitstream. It first estimates the available channel bandwidth through a hybrid model-based and probe-based 


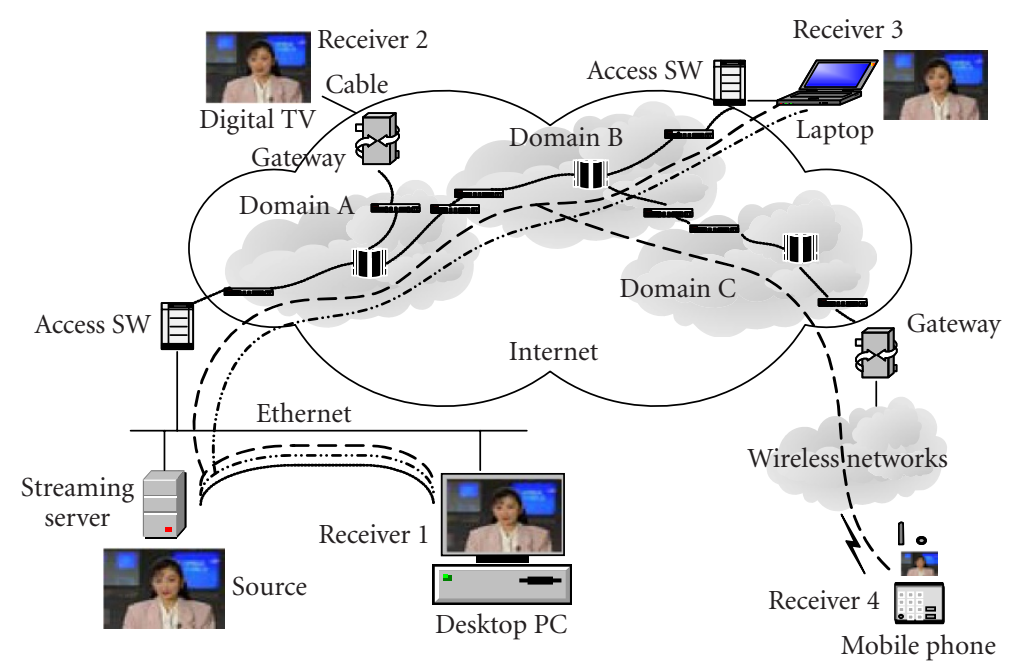

FIGURE 1: An exemplified scenario for streaming video.

method. Afterward, the transmitted video bitstreams are truncated to a bit rate that fits well in the estimated channel bandwidth. Since packet losses are inevitable in the general Internet, error control mechanism is a key component in this part. A flexible error resilience technique is proposed to adaptively enhance the robustness of SMART video bitstream. In addition, the SMART system provides a layered bitstream structure with a more important base layer and less important enhancement layers. Forward error correction (FEC) and automatic retransmission request (ARQ) techniques are applied to the base layer so as to reduce packet loss ratio and retransmission delay.

The rest of this paper is arranged as follows. Section 2 gives a brief overview of the SMART system. The SMART video coding techniques are discussed in Section 3. Section 4 introduces the channel estimation method used in the SMART system. The flexible error resilience technique and unequal error protection are described in Section 5. The experimental results presented in Section 6 demonstrate the advantages of the SMART system. Finally, Section 7 concludes this paper.

\section{OVERVIEW OF THE SMART SYSTEM}

This section gives an overview of the SMART coding and streaming system. At present, there are two modes for a streaming server to deliver video data to users: multicast and unicast. In the multicast mode, the server needs to send only one bitstream to a group of users, which is automatically replicated to all group members $[9,10]$, but this requests that the network has to be equipped with multicast-enable routers. In the unicast mode, the server delivers video bitstream to each user individually. The connection conditions between the server and each user can be estimated and monitored during transmission.

Since many routes in the current Internet do not enable the multicast mode, the SMART system discussed in this pa- per will focus on the unicast applications. Figure 2 illustrates the block diagram of the SMART system. Source video is first input into the SMART encoder module to generate a base layer bitstream and one or two enhancement layer bitstreams. Besides bitstreams, the SMART encoder generates a description file for each enhancement bitstream that contains all information for flexible error resilience and packetization. The detailed coding techniques will be discussed in Section 3, and the description file is introduced in Section 5. If the SMART encoder is powerful enough for real-time compression, the generated bitstreams can be directly packed and delivered just as in the live streaming applications. For the on-demand streaming applications, both the generated bitstreams and description files are saved in the storage device for future retrieval.

When the user submits a request to the SMART streaming server, like the real-time streaming protocol (RTSP) [11], the retrieved content, destination address, and user device capability are first transmitted by the transmission control protocol (TCP). After the control module in the SMART server receives the request, one user datagram protocol (UDP) connection is established immediately between the server and the user. Both the video data and the feedback from the SMART client are transmitted by this UDP connection. At the same time, the control module informs the server to retrieve the requested content from the storage device.

In the initial stage, the SMART system does not know the current channel conditions between the server and the client. Thus the base layer bitstream is packed with the real-time transport protocol (RTTP) [12] format using default channel parameters. At the same time, a prespecified FEC strategy is used in the base layer bitstream to generate parity packets. In general, since the base layer bit rate is very low in the SMART system, several seconds of source and parity packets can be rapidly delivered to the client as prebuffering. By transmitting these packets, the statistic channel parameters, such as packet loss ratio and latency, are packed with the 


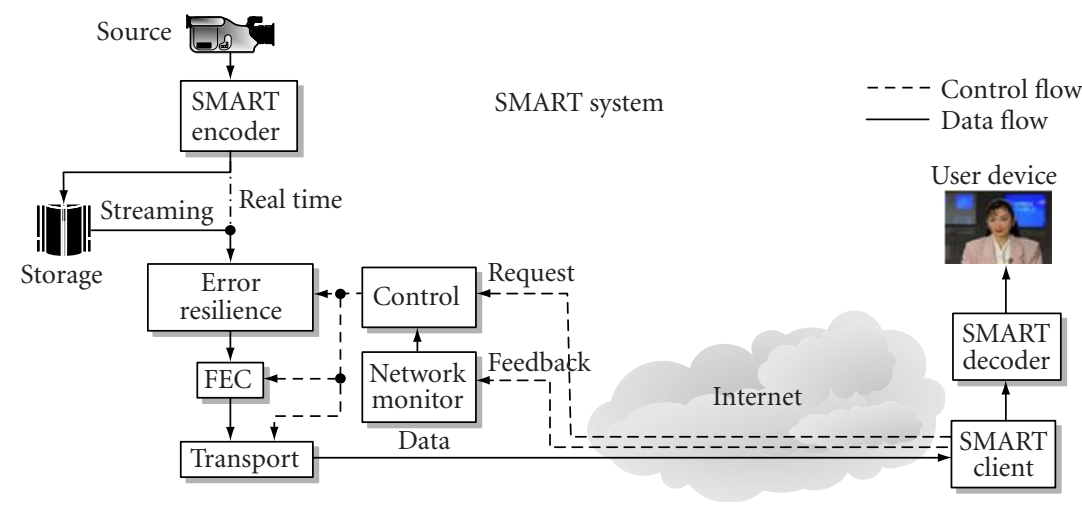

FIgURE 2: The block diagram of the SMART system.

real-time control protocol (RTCP) format [12] and sent back to the network monitor module in the SMART server. Accordingly, the SMART server can estimate the current available channel bandwidth.

With the obtained channel parameters, the SMART server starts to optimally pack the base layer and enhancement layer bitstreams with RTTP format. FEC protection depth to the base layer can be also adaptive to the channel conditions. In order to avoid network congestion, the actual bandwidth for the enhancement layer is the remaining part of the estimated channel bandwidth after delivering the base layer and FEC packets. Since the enhancement layer bitstream provides bit level scalability, it can be readily and precisely truncated to fit in the given bandwidth. Consequently, the SMART system can fully utilize available channel bandwidth and provide the user with better quality. Packet loss ratio and latency are periodically sent back by the client. The SMART server can timely adjust data transmission according to the feedbacks and the estimated channel bandwidth.

In the SMART system, another important feedback from the client is the negative acknowledgement (NACK) to notify the SMART server in which base layer packets are lost during transmission. Since the base layer is still a nonscalable bitstream, any lost packet would make the quality of the frames followed in the same GOP degrade rapidly. Therefore, the ARQ technique is also used to protect the base layer in the SMART system. Once the client detects lost packets at the base layer, a feedback is immediately sent out. The server will rapidly retransmit the lost packets. At the same time, any ARQ request received by the server will affect the sending rate to prevent further congestion in the channel. Since the base layer bit rate is very low in the SMART system, they can be strongly protected with small overhead bits. In addition, SMART video coding also provides the enhancement layer with an inherent error recovery feature. Any lost packet does not cause obvious visual artifacts. Moreover, it can be gracefully recovered in the following frames. Therefore, the current SMART system does not have any protection to the enhancement layer bitstreams.
In the following sections, the key techniques used in the SMART system, such as SMART video coding, bandwidth estimation, error resilience, and unequal error protection, will be discussed in detail.

\section{SMART VIDEO CODING}

How to efficiently compress video data with various scalabilities of rate, quality, temporal, spatial, and complexity is an active research topic in video coding field. Scalable video coding techniques have been developed rapidly in the past decade. Among them, spatial and temporal scalable coding techniques that provide video presentation at different resolutions, and frame rates have been accepted in some main video coding standards such as MPEG-2, MPEG-4, and H.263++ $[13,14,15]$.

In addition, FGS video coding techniques have been extensively studied in recent years. MPEG-4 standard already accepted the bit plane coding technique in the streaming video profile (SVP) $[16,17]$. In MPEG-4 FGS, an encoder using the motion-compensated discrete cosine transformation (DCT) transform coding generates a base layer video as the lowest quality layer. The residue between the original image and the reconstructed base layer image forms the enhancement layer with the bit plane coding technique, which provides an embedded bitstream and fine granularity quality and temporal scalabilities.

One major feature in MPEG-4 FGS is that the base layer and all the bit planes at the enhancement layer in a predicted frame are always compensated from the reconstructed version of the base layer in the reference. Therefore, it provides a remarkable capability in both bandwidth adaptation and error recovery. By predicting the enhancement layer from the base layer, any bitstream truncation and lost packets at the enhancement layer have no effect on the frames followed. However, this also makes MPEG-4 FGS suffer from severe degradation in coding efficiency due to the lowest quality reference. Furthermore, it is difficult for MPEG-4 FGS to compress different-resolution video at different layers; otherwise, 


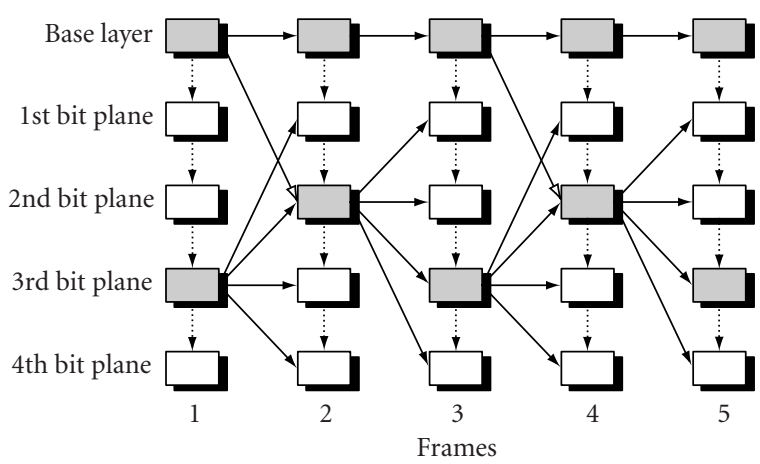

FIGURE 3: The proposed multiple-loop prediction technique with two references case.

the coding efficiency at the enhancement layer would be further degraded.

Therefore, the SMART video coding is proposed based on our previous works $[18,19]$. The multiple-loop prediction and drifting reduction techniques are first used at the quality enhancement layer to achieve a good trade-off between high coding efficiency and low drifting errors. Then, these techniques are extended to the temporal and spatial scalabilities, consequently, forming an efficient and universal scalable video coding framework.

\subsection{Multiple-loop prediction}

The multiple-loop prediction technique was first proposed in $[18,19]$ to improve the coding efficiency of MPEG-4 FGS. The basic idea is to use as many predictions from the enhancement layer as possible instead of always using the base layer as in MPEG-4 FGS. Because the quality of a frame is higher at the enhancement layer than at the base layer, this will make motion prediction more accurate, thus improving the coding efficiency. Considering the cost by introducing multiple references at the enhancement layer, Figure 3 illustrates a typical multiple-loop prediction scheme with one additional reference used in the enhancement layer coding.

In Figure 3, the gray rectangular boxes denote the reconstructed base layer or the reconstructed enhancement layer at a certain bit plane as references for the next frame coding. Solid arrows with solid lines between two adjacent frames are for temporal prediction, solid arrows with dashed lines are for prediction in the transform domain, and hollow arrows with solid lines are for reconstruction of high-quality reference from the previous base layer. Each frame at the base layer is always predicted from the previous frame at the base layer (low-quality reference) so as to avoid any effect from the lost enhancement data. Each frame at the enhancement layer is predicted from the previous frame at the enhancement layer (high-quality reference) for high coding efficiency.

In the FGS video coding schemes, the base layer bit rate is usually very low. It is reasonable to assume that the base layer bitstream can be completely transmitted to the client. Since the base layer is still predicted from the previous base layer, any bitstream truncation and lost packets at the enhance- ment layer have no effect on the base layer video. However, when those bit planes used to reconstruct the high-quality reference are truncated or corrupted during transmission, this would inevitably cause drifting errors at the enhancement layer. As a result, the decoded enhancement layer video may be deteriorated rapidly.

\subsection{Drifting reduction}

In order to effectively reduce drifting errors at the enhancement layer, the basic idea is to make sure that the encoder and the decoder have the same reconstructed reference for any future frame prediction, although the reconstructed reference may not have the best quality it could get if reconstructed using the high-quality reference.

We will show this idea through an example in Figure 3. In the decoder end, if the third bit plane in Frame 1 is truncated or dropped which is used in the encoder end to get the high-quality reference, the enhancement layer in Frame 2 will have to use the previous low-quality reference instead. Of course, some quality losses would be introduced by doing so. However, as long as in both the encoder end and the decoder end the reconstruction of the high-quality reference of Frame 2 always uses the base layer of Frame 1 as the reference, then the errors in Frame 1 could not further propagate to any frames followed. In other words, the reference used for prediction could be different from that used for reconstruction. This feature will prevent the errors drifting and preserve all the bandwidth adaptation and error recovery features as in MPEG-4 FGS.

As shown by hollow arrows with solid lines in Figure 3, some frames, such as Frames 2 and 4, reconstruct the highquality references from the previous low-quality reference at both the encoder and the decoder to prevent the errors propagating into future frames. However, if the third bit plane of Frame 1 is available at the decoder end, a better second bit plane quality of Frame 2 can still be reconstructed from the high-quality reference for display purpose only. In other words, the reconstruction of display image can be different from that of reference image.

Although the proposed technique significantly reduces the drifting errors from the previous frames, it still has a negative effect on coding efficiency because the high-quality reference does not always get the best quality it could get. If the reference for prediction and reconstruction is chosen as frame-based, that is, all enhancement layer macroblocks in a frame with the same reference, it is very difficult for the SMART video coding to provide a good trade-off between high coding efficiency and low drifting errors.

\subsection{Macroblock-based mode selection}

The technique choosing the proper reference for prediction and reconstruction at each enhancement layer macroblock is first proposed in [20]. Derived from MPEG-4 FGS and Figure 3, three intercoding modes as shown in Figure 4 are defined for coding the enhancement inter macroblock. The rectangular boxes in the first row denote the base layer and the rectangular boxes in other rows denote bit planes at the 


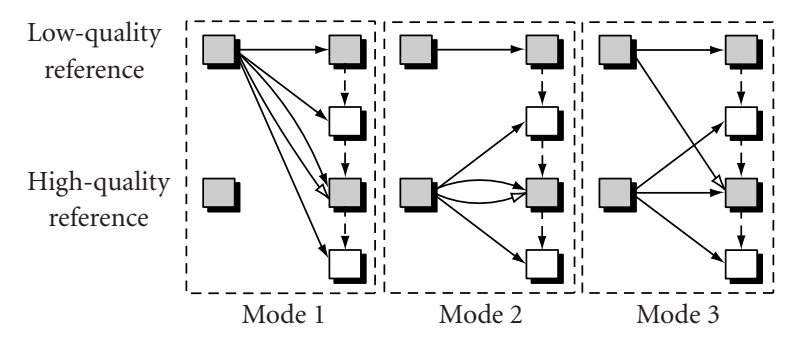

FIGURE 4: Three intercoding modes for the quality enhancement layer.

enhancement layer. Gray rectangular boxes indicate those to be reconstructed as references. Solid arrows with solid lines between two adjacent frames are for temporal predictions, solid arrows with dashed lines are for prediction in the transform domain, and hollow arrows with solid lines are for reconstruction of high-quality reference from the previous base layer.

In Mode 1, the base layer and the enhancement layer are both predicted and reconstructed from the previous lowquality reference. Since the low-quality reference is always available at the decoder, there is no drifting error in this mode. The coding efficiency of this mode is low due to lowquality temporal prediction. If all enhancement layer macroblocks are encoded with this mode, the proposed scheme is similar to MPEG-4 FGS.

In Mode 2, the base layer is predicted and reconstructed from the previous low-quality reference, but the enhancement layer is predicted and reconstructed from the previous high-quality reference. It can significantly improve the coding efficiency at moderate and high bit rates. There is no drifting error at the base layer. When the channel bandwidth is not high enough to transmit the high-quality reference, this mode would cause drifting errors at the enhancement layer.

In Mode 3, the enhancement layer is predicted from the previous high-quality reference while reconstructed from the previous low-quality reference at both the encoder and the decoder. This mode was for the purpose of drifting reduction. Since the low-quality reference is always consistent at both the encoder and the decoder, the drifting errors propagated from previous high-quality references can be eliminated with Mode 3.

More intercoding modes could be readily added in the SMART coding as long as they have the virtue in improving coding efficiency or reducing error propagation. In order to achieve a good trade-off between low drifting errors and high coding efficiency, a mode selection algorithm is proposed to choose the proper coding mode for each macroblock. Besides the above three intermodes, intramode is allowed in the enhancement layer coding. Intramode or intermode is determined by motion estimation. If a macroblock is encoded with the intramode at the base layer, the corresponding enhancement macroblock is also encoded with the intramode without temporal prediction. If a macroblock at the base layer is encoded with temporal prediction, the proposed mode selection algorithm has to determine which intercoding mode should be used at the corresponding enhancement macroblock.

The reference for prediction in Mode 1 is of low quality but the reference used in Mode 2 and Mode 3 is of high quality. If the absolute mean of the predicted DCT residues produced in Mode 1 is less than that in Modes 2 and 3, the current macroblock is coded using Mode 1; otherwise, the mode selection algorithm further determines the coding mode between Mode 2 and Mode 3. Both Modes 2 and 3 are predicted from the high-quality reference, the difference between them lies in the reference for reconstruction. In general, most of the enhancement macroblocks should be coded with Mode 2 for high coding efficiency. Mode 3 is used only when the drifting errors are more than a given threshold. In order to estimate the potential drifting errors at the encoder, the iterative drifting model proposed in [21] is given as follows:

$y(n)=\left\{\begin{array}{l}0, \quad n=1, \\ \operatorname{MC}_{n}\left(y(n-1)+\mathrm{DCT}^{-1}(X(n-1))\right), \quad N \geq n>1 .\end{array}\right.$

Here, $N$ is the total number of frames in a GOP, $\mathrm{MC}(\cdot)$ and $\mathrm{DCT}^{1}$ denote motion compensation and IDCT, respectively, $y(n-1)$ is the accumulative error propagated to the $(n-1)$ th frame, and $X(n-1)$ is DCT coefficients encoded in those bit planes for reconstruction of the high-quality reference in the $(n-1)$ th frame. With motion compensation, their sum forms the next drifting errors in the $n$th frame. If the estimated drifting error $y(n)$ is more than the given threshold, this macroblock is encoded with Mode 3; otherwise, this macroblock is encoded with Mode 2.

For the convenience of a better understanding of the proposed multiple-loop prediction, drifting reduction, and macroblock-based mode selection, Figure 5 illustrates an exemplified block diagram of the SMART decoder with quality scalability. There are two reference frames in the decoder. The first one is located in the base layer decoder and stored in the frame buffer 0 as a low-quality reference, while the second one is located in the enhancement layer decoder and stored in the frame buffer as a high-quality reference.

Only the low-quality reference is allowed in the reconstruction of the base layer in order to assure that no drifting error exists at this layer. The enhancement layer can use two different quality references for reconstruction. The enhancement bitstream is first decoded using bit plane variable length decoding (VLD) and mode VLD. The bit planes at the enhancement layer are categorized into a lower enhancement layer and a higher enhancement layer. Only the bit planes at the lower enhancement layer are used to reconstruct the high-quality reference. In Figure 5, $n(t)$ is the number of bit planes at the lower enhancement layer and $m(t)$ is the number of additional bit planes for the reconstruction of the display frame.

The decoded block-based bit planes are used to reconstruct the DCT coefficients of the lower and higher enhancement layers using the bit plane shift modules. After inverse 


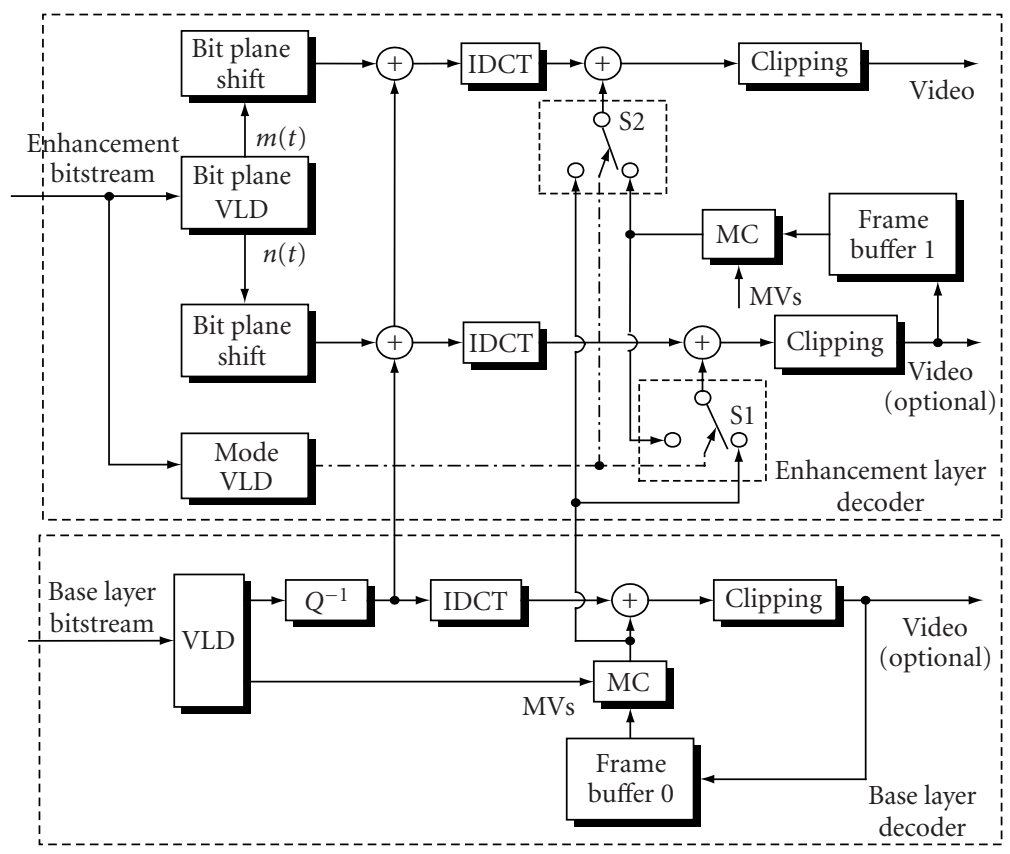

FIGURE 5: The exemplified SMART decoder with quality scalability.

DCT, the lower enhancement DCT coefficients plus the reconstructed base layer DCT coefficients generate the error image for reference, and all DCT coefficients including the higher enhancement layer generate the error image for display. Furthermore, there are two switches S1 and S2 at the SMART decoder that control which temporal prediction is used at each enhancement macroblock. The decoded macroblock coding mode decides the actions of the two switches. When one macroblock is coded as Mode 1, the switches S1 and S2 connect to the low-quality prediction. When it is coded as Mode 2, both of the switches S1 and S2 connect to the high-quality prediction. When it is coded as Mode 3, the switch $\mathrm{S} 1$ connects to the low-quality prediction. However, the switch S2 still connects to the high-quality prediction. Since the display frame does not cause any error propagation, the display frame is always reconstructed from the high-quality prediction in Mode 3.

\subsection{Universal scalable coding framework}

The techniques discussed in Sections 3.1, 3.2, and 3.3 can be readily extended to the temporal and spatial scalable video coding. The basic idea is to use more than one enhancement layer based on a common base layer to implement fine granularity quality, temporal, and spatial scalabilities within the same framework. In order to achieve high coding efficiency for various scalabilities, multiple prediction loops with different quality references are employed in the proposed framework. For example, by utilizing the high-quality reference in the spatial enhancement layer coding, the proposed framework can likewise fulfill efficient spatial scalability. The complexity scalability is inseparable with other scalabilities in the SMART codec. It is achieved by increasing/decreasing the bit rate, frame rate, and resolution. The changes in the frame rate and resolution provide coarse scalability on complexity. Because of the property of fine granularity of each layer on bit rate, the SMART codec also provides fine scalability on complexity by adjusting the bit rate of each layer. The lowest complexity bound is the lowresolution base layer decoding, which should be sufficiently low for many applications.

Figure 6 illustrates the proposed universal scalable coding framework. Source video with two resolutions is compressed in the proposed framework. Narrow rectangles denote low-resolution video and wide rectangles denote highresolution video. There are two different enhancement layers sharing a common base layer, and two optional enhancement ones. The bottom layer is the base layer. It is usually generated as the lowest quality, lowest resolution, least smoothness, and least complexity. The quality enhancement layer compresses the same resolution video as that at the base layer. It will improve the decoded quality of the base layer. The temporal enhancement layer improves the base layer frame rate and makes the decoded video look smooth. The rest two enhancement layers improve the video quality and frame rate at high resolution. These two enhancement layers are optional in the proposed framework and appear only if the video with two different resolutions is encoded. The same resolution enhancement layers are stored in the same bitstream file. Therefore, the SMART coding scheme generates at most three bitstreams: one base layer bitstream and two enhancement layer bitstreams.

Except that the base layer is encoded with the conventional DCT transform plus VLC technique, all of the enhancement layers are encoded with the bit plane coding technique. In other words, every enhancement layer bitstream can be arbitrarily truncated in the proposed framework. In 


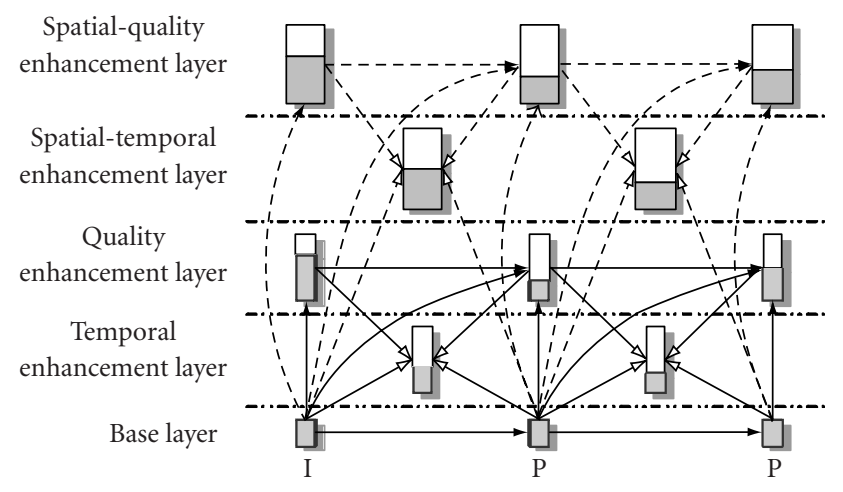

FIGURE 6: The proposed SMART coding framework.

Figure 6, each rectangle denotes the whole frame bitstream at one enhancement layer. The shadow region is the actual transmitted part, whereas the blank region is the truncated part. Hence the proposed SMART video coding provides the most flexible bit rate scalability.

Since the multiple-loop prediction technique is used in the proposed framework, every layer, excluding the base layer, can select the prediction from two different references. As shown by solid arrows with solid lines in Figure 6, the quality enhancement layer use the reconstructed base layer and the reconstructed quality enhancement layer at a certain bit plane as references. As shown by hollow arrows with solid lines, the temporal enhancement layer is bidirectionally predicted from the base layer and the quality enhancement layer. The predictions for the two high-resolution enhancement layers are denoted by solid arrows with dashed lines and hollow arrows with dashed lines, respectively.

Similarly, some intercoding modes are defined at the temporal and spatial enhancement layers, which can be found in $[22,23,24]$. Each coding mode has its unique references for prediction and reconstruction. The similar mode selection algorithm discussed in Section 3.3 can be also applied to the temporal and spatial enhancement layers. In fact, some other techniques proposed in $[25,26,27,28]$ can be easily incorporated into the framework by defining several new coding modes.

\section{CHANNEL ESTIMATION}

In the streaming applications, one important component is congestion control. Congestion control mechanisms usually contain two aspects: estimating channel bandwidth and regulating the rate of transmitted bitstream. Since the SMART video coding provides a set of embedded and full scalable bitstreams, rate regulation in the SMART system is essentially equal to truncating bitstreams to a given bit rate. There is not any complicated transcoding needed. The remaining problem is how to estimate the channel bandwidth.

Typically, channel estimation techniques are divided into two categories: probe-based and model-based. The probebased techniques estimate the channel bandwidth bottleneck by adjusting the sending rate in a way that could maintain packet loss ratio below a certain threshold [29]. The modelbased techniques are based on a TCP throughput model that explicitly estimates the sending rate as a function of recent packet loss ratio and latency. Specifically, the TCP throughput model is given by the following formula [30]:

$$
\lambda=\frac{1.22 \times \mathrm{MTU}}{\mathrm{RTT} \times \sqrt{p}},
$$

where $\lambda$ is the throughput of a TCP connection (in $\mathrm{B} / \mathrm{s}$ ), MTU is the packet size used by the connection (in bytes), RTT is the round-trip time of the connection (in seconds), and $p$ is the packet loss ratio of the connection.

With formula (2), the server can estimate the available bandwidth by receiving feedback parameters RTT and $p$ from the client.

Among all existing model-based approaches, TCPfriendly rate control (TCP-FRC) [31] is the most deployable and successful one. The sending rate formula, by considering the influence of time out, is given as follows:

$$
\lambda=\frac{\mathrm{MTU}}{\operatorname{RTT} \sqrt{2 p / 3}+\operatorname{RTO}(3 \sqrt{3 p / 8}) p\left(1+32 p^{2}\right)},
$$

where RTO is the TCP retransmission time-out value (in seconds).

However, TCP-FRC has one obvious drawback undesirable for the SMART system, that is, the estimated bandwidth always fluctuates periodically even if the channel bandwidth is very stable. The reason is that TCP-FRC is trying to increase the sending rate when there is no lost packet. This unfortunately leads to a short-term congestion. Since TCP-FRC is very sensitive in the low packet loss ratio case, the sending rate is greatly reduced again to avoid further congestion.

Therefore, the SMART system adopts a hybrid modelbased and probe-based method to estimate the available channel bandwidth. TCP-FRC is first used to calculate an initial estimated bandwidth by packet loss ratio and RTT. If there is no lost packet, the estimated bandwidth should be more than the previous estimation. On the other hand, some packets that contain less important enhancement data are transmitted with the probing method. This is a feature of the SMART bitstream. Even though those packets are lost for probing, they do not affect other data packets. In general, the estimated bandwidth by the probing method is viewed as the bottleneck between the server and the client. The estimated bandwidth in TCP-FRC should be not more than that estimated by the probing method. Therefore, the probing method provides an upper bound for TCP-FRC so as to reduce fluctuations in bandwidth estimation.

Video packets in the SMART system are categorized into three priorities for bandwidth allocation. The retransmitted and base layer packets have the highest priority. Estimated bandwidth is first used to deliver them to the client. The FEC packets of the base layer have the second priority. If the estimated bandwidth is more than that needed by the highest priority packets, they are delivered prior to the enhancement packets. Finally, the remaining channel bandwidth is 
used to deliver the truncated enhancement bitstreams. In fact, the enhancement packets also implicates several different priorities, For example, the bit planes for reconstruction of the high-quality reference are more important than other bit planes, and at low bit rates, the quality enhancement layer may be more important than the temporal enhancement layer, and so on. Because of limitation in pages, this paper no longer further discusses this issue.

\section{ERROR CONTROL}

In the streaming applications, error control mechanism is another important component to ensure received bitstreams decodable, which often includes error resilience, FEC, ARQ, and even error concealment $[32,33]$. In this section, we will discuss the error resilience technique and unequal error protection used in the SMART system.

\subsection{Flexible error resilience}

Packet losses are often inevitable while transmitting compressed bitstreams over the Internet. Besides the necessary frame header, some resynchronization markers and related header information have to be inserted in the bitstream generation so that the lost packets do not affect other data packets. This is the most simple error resilience technique, but very useful. The resynchronization marker plus the header and data followed is known as a slice. In MPEG-4, the resynchronization marker is a variable length symbol from 17 bits to 23 bits [14]. The slice header only contains the index of the first macroblock in this slice. In general, the resynchronization marker and the slice header are inserted at a given length or number of macroblocks.

However, this method has two obvious problems when it is applied to the enhancement layer bitstream in the SMART system. Firstly, although the SMART enhancement layer bitstream provides bit level scalability, the actual minimum unit in the packetization process is a slice. This would greatly reduce the granularity of scalability. Secondly, the slice length is decided in the encoding process and fixed in the generated bitstream. For the streaming applications, it is impossible to adjust the slice length again to adapt to channel conditions. In general, longer slice means lower overhead bits and bigger effects of lost packet. On the contrary, shorter slice means higher overhead bits and lower effects of lost packet. Adaptively adjusting the slice length is a very desirable feature in the streaming applications. Therefore, a flexible error resilience technique is proposed in the SMART enhancement layer bitstream.

In the SMART system, there are no resynchronization markers and slice headers in the enhancement layer bitstream. Thus, the generated bitstream is exactly the same as that without error resilience. But the positions of some macroblocks and their related information needed in the slice header are recorded in a description file. Besides the index of the first macroblock, the slice header at the enhancement layer also contains the located bit plane of the first macroblock. We call these macroblocks resynchronization points. Note that each resynchronization point is always macroblock

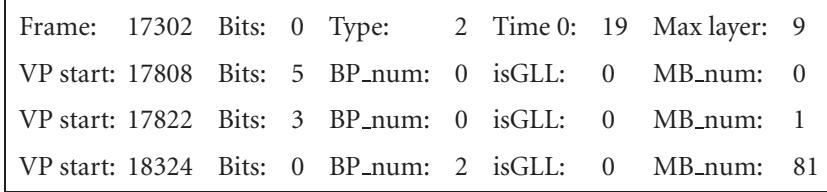

FIGURE 7: The exemplified description file.

aligned. In this stage, resynchronization points do not cause actual overhead bits in the generated bitstreams. Thus, the description file could even record every macroblock.

Figure 7 exemplifies the structure of the description file. The fields Frame and Bits in the same row are used to locate the start position of a frame in the bitstream. The units of these two fields are byte and bit, respectively. The field Bits is always zero in the first row of every frame due to bytealigned. The field Type indicates the frame type: 0 for I frame, 1 for $\mathrm{P}$ frame, and 2 for $\mathrm{B}$ frame. The field time is the relative time of the current frame. The first digit in this field denotes the number of seconds, and the second digit denotes the frame index in a second. The field Max Layer is the maximum number of bit planes in a frame. The fields VP start and Bits are used to locate the start position of a macroblock. The field BP_num is the located bit plane of the current macroblock. The field is GLL indicates whether this macroblock is used to reconstruct the high-quality reference or not. It provides a priority to transmit the enhancement bitstreams. The field $M B_{-}$num is the first macroblock index in a slice.

The proposed flexible error resilience is used only at the enhancement DCT data. If the motion vectors exist at the enhancement layer, for example, in temporal frames, they are differentially coded together before DCT coefficients. The VOP header and coded motion vectors are processed as a slice. There is not any resynchronization point within them in case that the lost motion vectors in a slice affect other motion vectors decoded in another slice due to motion vector prediction. Similar to the entropy coding used in MPEG4 FGS, there is not any DC and/or AC coefficient prediction among neighboring blocks. Therefore, the slices in a frame have no dependency except for the inherent relationship among bit planes.

With the description file, the proposed error resilience technique in the SMART system can choose any resynchronization points to chop an enhancement layer bitstream into slices. However, since the position of the resynchronization point may be not byte-aligned in the bitstream, one lost packet probably makes many packets followed undecodable. As showed in Figure 8, macroblock $N$ is a resynchronization point. It shares byte $m$ in the bitstream with macroblock $N-1$. If the macroblock $N$ is selected as the start of a slice, these two macroblocks may not locate in the same transport packet. If byte $m$ belongs to the previous packet, the packet of macroblock $N$ is even received undecodable when the packet of macroblock $N-1$ is lost during transmission.

A simple technique is proposed to solve this problem as shown in Figure 8. When a resynchronization point is selected as the start of one slice, the first byte of this macroblock 


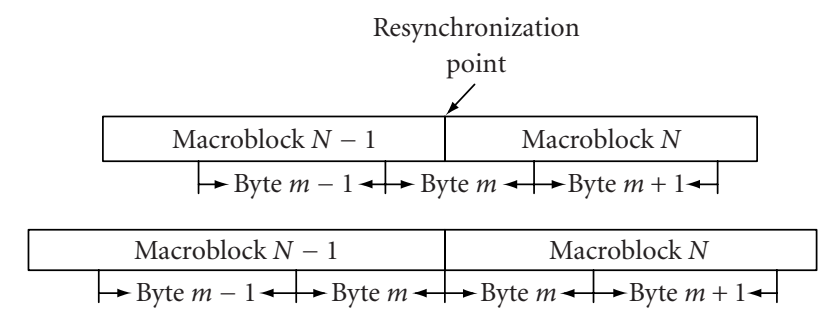

FIgURE 8: The error resilience in the SMART system.

is duplicated into two slices so that the lost packet cannot affect each other. This leads to the probability that the head and tail of each slice may have several useless bits. The decoder has to know how many useless bits should be skipped. Therefore, the numbers of useless bits in the head and tail generated from the description file need to be encapsulated into the transport packet and transmitted to the client. The fields MB_num and BP_num at the slice header also need to be encapsulated into the transport packet and transmitted to the client.

We evaluate the proposed error resilience technique compared with that in MPEG-4. In the proposed technique, a byte has to be duplicated for every selected resynchronization point. In addition, the corresponding numbers of useless bits are also contained in the packet. But, bits for the resynchronization marker in MPEG-4 bitstream can be saved. Therefore, the proposed technique has the similar overhead bits in each slice. However, it enables the SMART system to adaptively adjust the slice length according to rate-distortion optimization and channel conditions. This is a very desirable feature in the streaming applications.

\subsection{Unequal error protection}

Since the SMART video coding provides a layered bitstream structure with a more important base layer and less important enhancement layers, error protection techniques such as FEC and ARQ are unevenly applied to the base layer and the enhancement layer.

In general, if the streaming systems have no request on delay, FEC would not play an important role because the lost packets can be recovered by ARQ. In the SMART system, the bit rate of the base layer is very low and it may only occupy a small part of the total bit rate (usually less than 20\%). When four data packets are protected by one FEC packet, the overhead for FEC is only about 5\%. In return, if the lost packets take place randomly, most of them may be recovered by FEC. It will considerably reduce the system delay due to ARQ. Based on these considerations, the SMART system uses FEC as an option at the base layer if low delay is requested in some applications. It also provides a space to achieve a better tradeoff between ARQ delay and FEC overhead.

When FEC is enabled, the base layer packets are divided into many groups containing $K$ source packets per group. Assume that $N-K$ parity packets will be produced with a Reed-Solomon codec. When these $N$ packets are transmitted over the best-effort Internet, any received subset of $K$ source or parity packets can be used to reconstruct the original $K$ source packets. In the SMART system, $K$ is often set as $N-1$ in order to avoid too much overhead introduced by FEC. The target using FEC is mainly to recover occasional lost packet and reduce the delay caused by ARQ.

The base layer bitstream in the SMART system is a nonscalable one. Furthermore, the motion compensation technique is used in the base layer coding. Any lost packet will make the quality of the frames followed in a GOP degrade rapidly. Therefore, the ARQ technique is also applied to the base layer to handle burst packet losses. If the lost packets that cannot be recovered from FEC are detected at the base layer, a NACK feedback is immediately sent to the server. If no acknowledgement feedback is received, the transmitted base layer packets are saved in a special buffer. The SMART will get the lost base layer packets from the special buffer and retransmit them to the client until time out. If the base layer packets arrive too late or are not able to be recovered by FEC and ARQ, the SMART system will skip to the next GOP. In addition, the client periodically sends the acknowledgement feedback so that the server discards the received base layer packets from the special buffer.

From the discussions in Section 3, we know that the SMART video coding provides the embedded enhancement bitstreams. Any truncation and lost packets at the enhancement bitstream are allowed. It can be gracefully recovered by the drifting reduction technique. Therefore, no error protection techniques are applied to the enhancement packets in the current SMART system. In fact, consider that the lost packets in low bit planes used to reconstruct the high-quality reference may still have a big effect on maintaining high decoded quality. The techniques for partly protecting the enhancement layer packets should be further investigated.

\section{EXPERIMENTS}

Both static and dynamic experiments are designed to evaluate the performances of the SMART system on coding efficiency, channel estimation, bandwidth adaptation, error robustness, and so on.

\subsection{Static tests}

Three different coding schemes, namely, MPEG-4 FGS without global motion compensation, SMART coding without multiple-loop prediction, and SMART coding, are compared in terms of coding efficiency. MPEG-4 FGS provides the reference of scalable coding scheme for comparisons. The final drift amendment (FDAM) software of MPEG-4 FGS released in June 2002 is used to create the results of MPEG-4 FGS [34]. The SMART system uses Windows Media Video Encoder 8.0 (WMV8) as the base layer codec. The MPEG-4 testing sequences Foreman and Coastguard with common intermediate format (CIF) are used in this experiment.

In the first set of experiments, the testing sequences are coded at $10 \mathrm{~Hz}$ encoding frame rate. Only the first frame is encoded as I frame and the rest of the frames are encoded as $\mathrm{P}$ frames. The main parameters in the MPEG-4 FGS base layer are given as follows: 


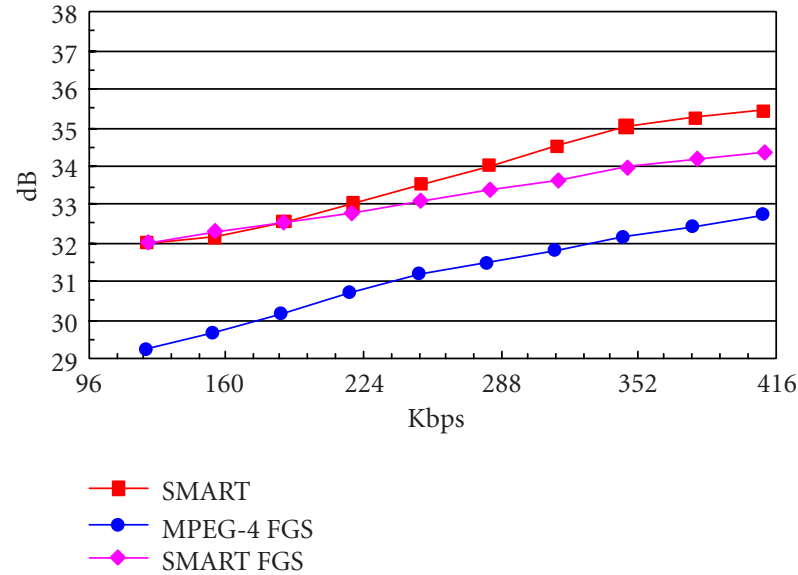

(a)

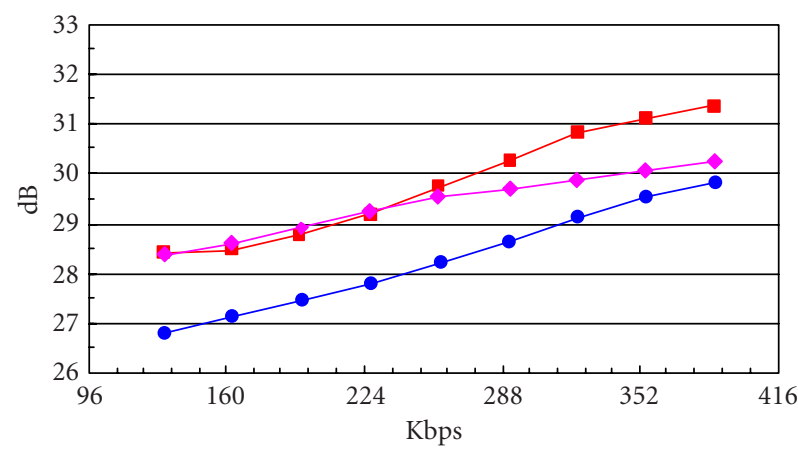

$\longrightarrow$ SMART
$\because$ MPEG-4 FGS
$\longrightarrow$ SMART FGS

(b)

FIGURE 9: The curves of average PSNR versus bit rate at $10 \mathrm{fps}$ without B frame and temporal scalability. (a) Foreman CIF $Y(10 \mathrm{~Hz})$. (b) Coastguard CIF Y $(10 \mathrm{~Hz})$.

(i) motion estimation: \pm 32 pixels,

(ii) motion compensation: quarter-pixel precision,

(iii) quantization: $M P E G$,

(iv) direct search range: 2 (half-pixel unit),

(v) advanced prediction: Enable,

(vi) skipped macroblock: Enable.

The results of the first set of experiments are depicted in Figure 9. In the curves of MPEG-4 FGS, the base layer is coded with quantization parameter 31 , and the quality enhancement layer bitstream is truncated at an interval of $32 \mathrm{kbps}$. By adjusting the quantization parameter, the SMART curve has a bit rate at the base layer similar to MPEG-4 FGS. The curves of SMART FGS are obtained with the SMART system by only using Mode 1. The curves of SMART are obtained with all the discussed coding techniques in this paper.

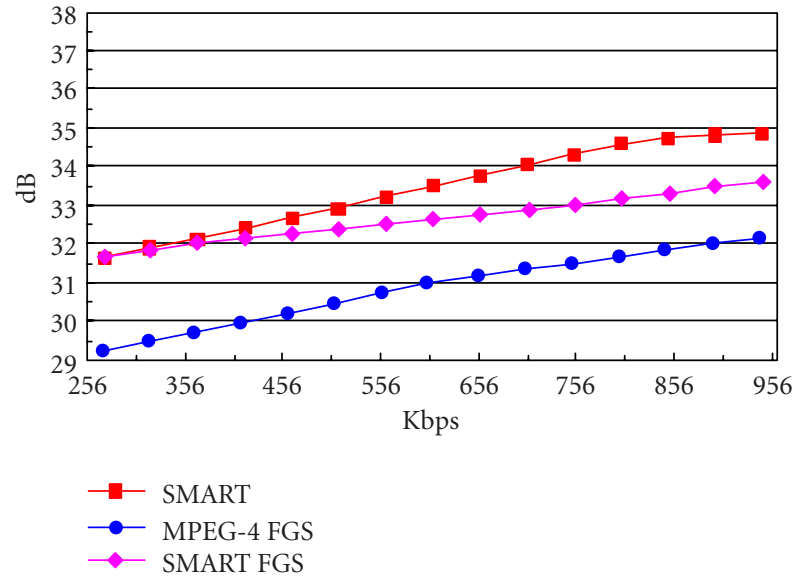

(a)

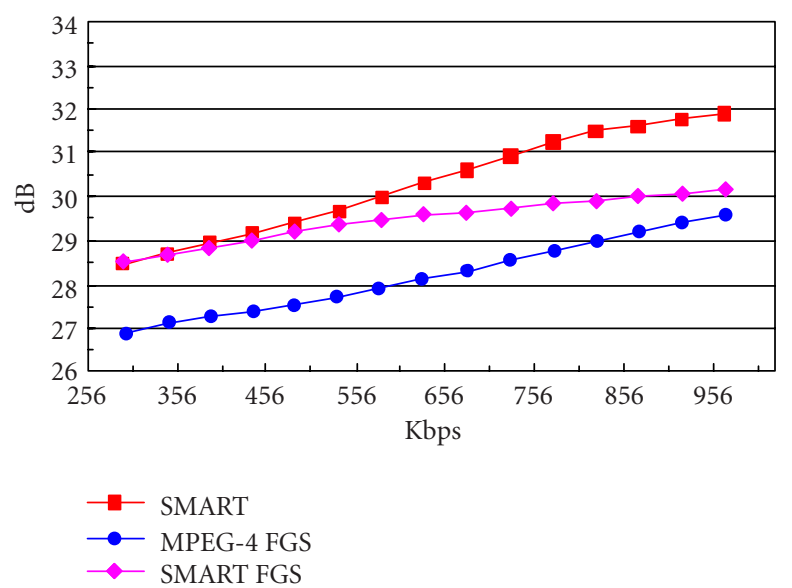

(b)

FIGURE 10: The curves of average PSNR versus bit rate at $30 \mathrm{fps}$ with B frame and temporal scalability. (a) Foreman CIF $Y(30 \mathrm{~Hz})$. (b) Coastguard CIF Y $(30 \mathrm{~Hz})$.

SMART FGS and SMART use the same coding technique at the base layer. Since only Mode 1 is used in SMART FGS, the enhancement layer coding is essentially the same as that in MPEG-4 FGS. WMV8 provides a very good base layer compared with MPEG-4; the coding efficiency gain at the base layer is close to $2.8 \mathrm{~dB}$ in Foreman and $1.6 \mathrm{~dB}$ in Coastguard compared with MPEG-4 FGS. But without the proposed enhancement prediction technique, the coding efficiency gain is becoming smaller and smaller with bit rates increasing. The coding efficiency gain of SMART FGS is only $1.6 \mathrm{~dB}$ in Foreman and $0.44 \mathrm{~dB}$ in Coastguard at the highest bit rate. However, the SMART curves with the proposed techniques present the consistent performance in a wide range of bit rates. The bit rate for the high-quality reference is about 346 kbps in Foreman and 322 kbps in Coastguard. The coding efficiency gain, when the high-quality reference is available, is $2.9 \mathrm{~dB}$ in Foreman and $1.7 \mathrm{~dB}$ in Coastguard. 


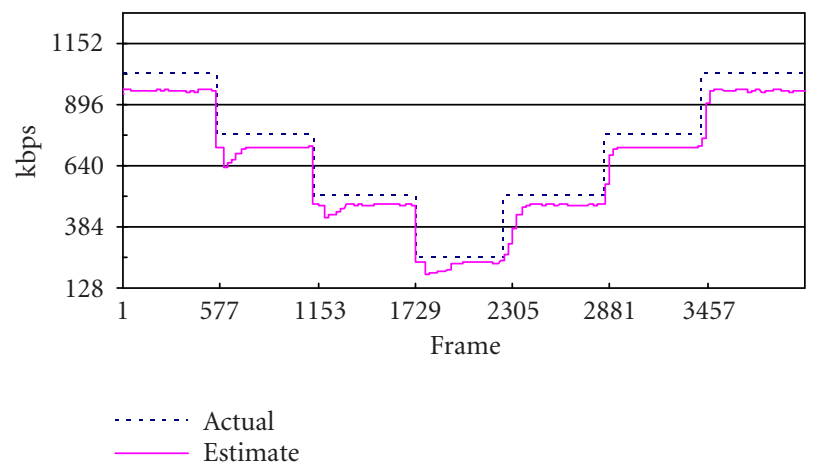

(a)

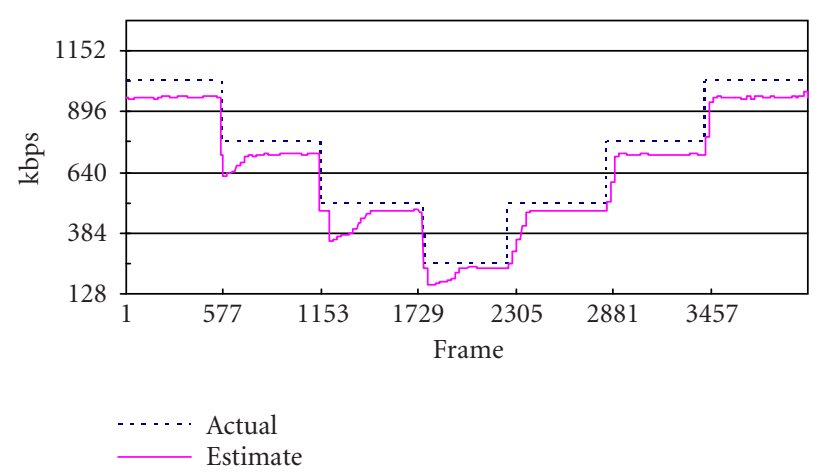

(b)

FIgURE 11: The estimated channel bandwidth in the SMART system. (a) Estimated bandwidth in bs_one sequence. (b) Estimated bandwidth in bs_two sequence.

In addition, although the high-quality references are used in the enhancement layer coding, the SMART curves still have the similar performance as the SMART FGS curves at low bit rates. The SMART curve has only about $0.15 \mathrm{~dB}$ loss at $150 \mathrm{kbps}$. This proves that the proposed drifting reduction technique can effectively control the drifting errors.

In the second set of experiments, the testing sequences are coded at $30 \mathrm{~Hz}$ encoding frame rate. Only the first frame is coded as I frame. There are two temporal frames in the scalable coding scheme between a pair of I and P or two P frames. Other experimental conditions are the same as in the first set of experiments. The same experimental results given in Figure 10 are observed as in the first set of experiments.

Since neither MPEG-4 FGS nor the SMART codec contains one of the switching techniques, for example, $S$ frame, SP frame, or SF frame, the readers who are interested in the comparisons between the scalable video coding and the SP frame on H.26L TML can read the MPEG proposal in [35].

\subsection{Dynamic tests}

The dynamic experiments try to test the SMART system under the dynamic channel, such as streaming video over the Internet, where the channel bandwidth varies in a wide range of bit rates. The conditions of MPEG-4 FGS verification test

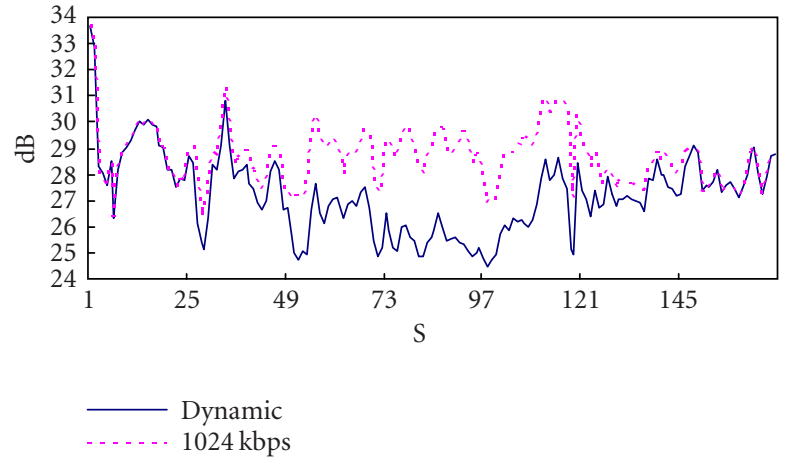

(a)

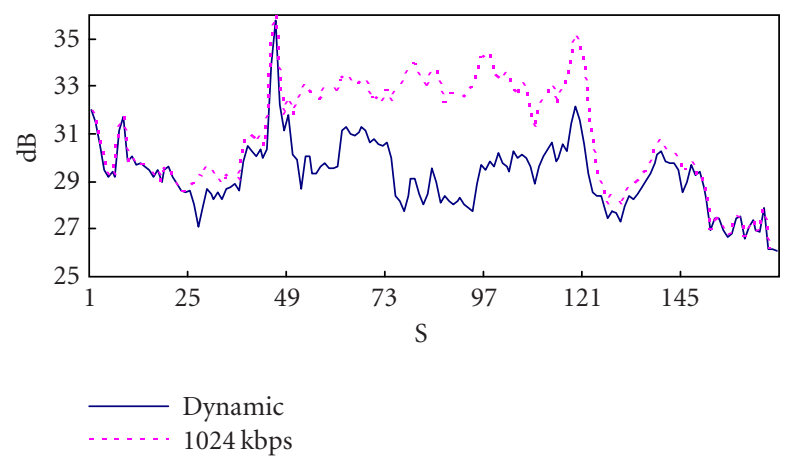

(b)

Figure 12: The decoded quality over the dynamic channel: (a) bs_one $Y$. (b) bs_two $Y$.

are used in this experiment [36]. Two CIF sequences, bs_one and bs_two, each with 4032 frames (168 seconds at $24 \mathrm{fps}$ ) are used. The channel bandwidth varies from $1024 \mathrm{kbps}$ to $256 \mathrm{kbps}$ and then recovers to $1024 \mathrm{kbps}$ again with a step of 256 kbps. Every bit rate lasts 24 seconds. The dynamic channel simulation is done by the commerce simulator, the Cloud software (http://www.shunra.com).

By using the hybrid model-based and probe-based bandwidth estimation scheme, when the sequences bs_one and bs_two are transmitted over the simulated dynamic channel, the estimated bandwidth is recorded and plotted in Figure 11. The dashed-line curves are the actual channel bandwidth limited by the Cloud simulator. When the channel bandwidth switches from high bit rate to low bit rate, the estimated bandwidth with TCP-FRC can rapidly decrease in order to avoid network congestion. When the channel bandwidth increases, the estimated bandwidth can also catch this variation at a short time. Furthermore, the curves in Figure 11 fully demonstrate the advantage of the hybrid bandwidth estimation method, where the probing method gives an upper bound to prevent TCP-FRC from raising the sending rate over the network bottleneck. Therefore, the SMART system has a stable estimation when the channel bandwidth stays in a constant. 
The decoded quality of sequences bs_one and bs_two are also recorded and plotted in Figure 12. Each sample is the average PSNR in a second. Two factors, channel bandwidth and video content, will affect the final decoded quality. Sometimes, even if the channel bandwidth is high, the decoded PSNR may not be high due to active content. In order to eliminate the video content factor in evaluating the performance of the SMART system on bandwidth adaptation, the PSNR curves decoded at $1024 \mathrm{kbps}$ are drawn in Figure 12 as reference. The distances between the dynamic curve and the $1024 \mathrm{kbps}$ curve reflect the bandwidth adaptation capability of the SMART system.

As shown in Figure 12, the decoded PSNR is less than that at $1024 \mathrm{kbps}$ up to $4.4 \mathrm{~dB}$ from 73 to 96 seconds because the estimated bandwidth is only $240 \mathrm{kbps}$ around. From 49 to 72 seconds and from 97 to 120 seconds, the estimated channel bandwidth is about $480 \mathrm{kbps}$. The decoded PSNR is significantly improved compared with that at $240 \mathrm{kbps}$. From 25 to 48 seconds and from 121 to 144 seconds, the estimated bandwidth is about $720 \mathrm{kbps}$. The decoded PSNR is only slightly less than that at $1024 \mathrm{kbps}$. The SMART system provides almost the same quality as that at $1024 \mathrm{kbps}$ from 1 to $24 \mathrm{sec}-$ onds and from 145 to 168 seconds. The estimated bandwidth in these two periods is about $950 \mathrm{kbps}$. Thus, the SMART system shows excellent performance on bandwidth adaptation.

Although there are a lot of packet losses while switching the channel bandwidth from high bit rate to low bit rate, with the proposed error resilience technique and unequal error protection, all packet losses at the base layer are recovered in the simulation. No green blocks appeared in the decoded video. For the enhancement bitstreams, there is not any error protection. The effects of packet losses at the enhancement layer are gradually recovered by the drifting reduction technique. There are also no obvious visual artifacts and quality degradation in the average PSNR curves.

At last, the SMART video player is given in Figure 13. It can real-time decode the CIF sequence at $1024 \mathrm{kbps}$ with PIII $800 \mathrm{MHz}$. The decoded video is presented in the biggest window. The right-upper window shows the curve of the estimated channel bandwidth and the right-bottom window is for the program list. The packet loss ratio is drawn in the window between them. A progress bar is used to indicate the status of the received buffer.

The proposed SMART system is also used to run the results of MPEG-4 FGS verification tests, where the SMART codec is replaced by MPEG-4 FGS codec. The experimental results have been released in [37].

\section{CONCLUSIONS AND FUTURE WORKS}

The SMART system presents an efficient, adaptive, and robust scheme for streaming video over the Internet. Firstly, since the multiple-loop prediction and drifting reduction techniques are applied at the macroblock level, the SMART system can outperform MPEG-4 FGS up to $3.0 \mathrm{~dB}$. Secondly, the SMART system has excellent capability in network bandwidth and device adaptation due to the embedded enhance-

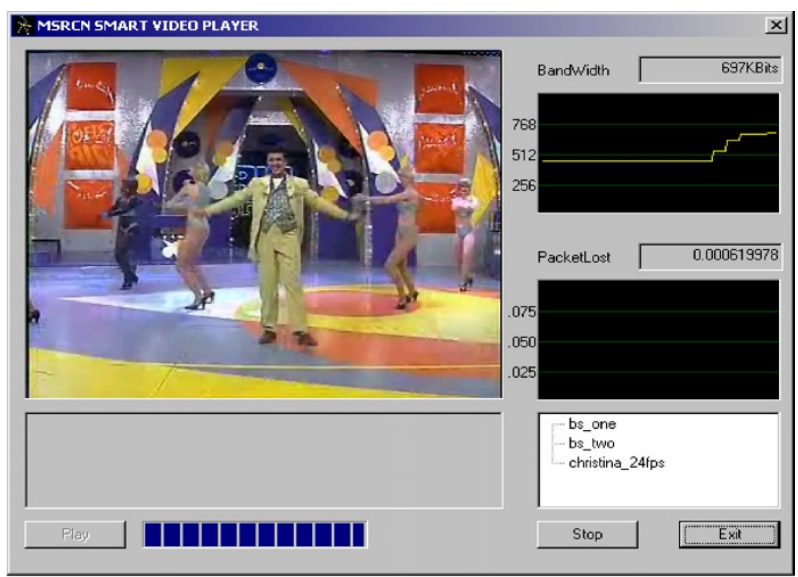

FIgUre 13: The interface of the SMART video player.

ment bitstreams and the universal scalabilities. Thirdly, with the proposed bandwidth estimation method, the SMART system can rapidly and stably catch bandwidth variations. At last, since a layered bitstream structure with a more important base layer and less important enhancement layers is provided in the SMART system, the base layer bitstream is highly protected by the proposed error resilience and unequal error protection techniques with small overhead. The SMART system can provide users with much smooth playback experience and much better visual quality in the best-effort Internet.

Although the SMART system shows good performances on coding efficiency, bandwidth adaptation, channel estimation, and error robustness, there are still several problems needed to be further studied in the future, such as how to further improve the coding efficiency to cover an even wider bit rate range; how to optimally allocate the available bandwidth to different enhancement layers so that the perception quality looks better; how to optimally packetize the base layer and the enhancement layer bitstreams so that the packet losses have less effects; how to optimally decide the parameters in FEC and ARQ to achieve a better trade-off between ARQ delay and FEC overhead; and how to protect those bit planes for reconstruction of the high-quality reference at the enhancement layers with small overhead. In addition, how to effectively utilize the features and techniques of the SMART system in the multicast applications is another topic worthy of further study.

\section{ACKNOWLEDGMENTS}

Many colleagues and visiting students in Microsoft Research Asia also took part in the SMART system. The authors would like to thank Dr. W. Zhu, Dr. Q. Zhang, and L. Wang for their contribution in the bandwidth estimation part; X. Sun for fine-grain quality and temporal scalability; Dr. Q. Wang and Dr. R. Yang for fine-grain spatial scalability; and Prof. Z. Xiong and S. Cheng for setting up the SMART server in Texas A\&M University. 


\section{REFERENCES}

[1] J. Lu, "Signal processing for Internet video streaming: a review," in Proc. SPIE: Image and Video Communications and Processing 2000, vol. 3974, pp. 246-259, San Jose, Calif, USA, January 2000.

[2] A. Luthra, Need for simple streaming video profile, ISO/IEC JTC1/SC29/WG11, M5800, Noordwijkerhout, The Netherlands, March 2000.

[3] D. Wu, Y. T. Hou, W. Zhu, Y.-Q. Zhang, and J. M. Peha, "Streaming video over the Internet: approaches and directions," IEEE Trans. Circuits and Systems for Video Technology, vol. 11, no. 3, pp. 282-300, 2001.

[4] RealNetworks facts, 2001, http://www.realnetworks.com/ company.

[5] Windows Media technologies, http://www.microsoft.com/ windows/windowsmedia.

[6] N. Farber and B. Girod, "Robust H.263 compatible video transmission for mobile access to video servers," in Proc. International Conference on Image Processing, vol. 2, pp. 73-76, Santa Barbara, Calif, USA, October 1997.

[7] M. Jarczewicz and R. Kurceren, A proposal for SP-frames, ITU-T Q.6/SG 16, VCEG-L27, Elysee, Germany, January 2001.

[8] X. Sun, S. Li, F. Wu, G. B. Shen, and W. Gao, "Efficient and flexible drift-free video bitstream switching at predictive frames," in Proc. IEEE International Conference on Multimedia and Expo, vol. 1, pp. 541-544, Lausanne, Switzerland, August 2002.

[9] S. McCanne, V. Jacobson, and M. Vetterli, "Receiver-driven layered multicast," in Proc. Conference on Applications, Technologies, Architectures, and Protocols for Computer Communication (ACM SIGCOMM '96), pp. 117-130, Stanford, Calif, USA, August 1996.

[10] D.-N. Yang, W. Liao, and Y.-T. Lin, "MQ: an integrated mechanism for multimedia multicasting," IEEE Trans. Multimedia, vol. 3, no. 1, pp. 82-97, 2001.

[11] H. Schulzrinne, A. Rao, and R. Lanphier, Real time streaming protocol (RTSP), Internet Engineering Task Force, Internet draft, RFC 2326, April 1998.

[12] H. Schulzrinne, S. Casner, R. Frederick, and V. Jacobson, RTP: A transport protocol for real-time applications, Internet Engineering Task Force, Internet draft, RFC 1889, January 1996.

[13] MPEG video group, Information technology-Generic coding of moving pictures and associated audio, ISO/IEC 13818-2, International standard, 1995.

[14] MPEG video group, Generic coding of audio-visual objects: part 2, ISO/IEC 14496-2, International standard, 1998.

[15] ITU-T Recommendation H.263, Video coding for low bit rate communication, Version 2, 1998.

[16] W. Li, "Overview of fine granularity scalability in MPEG-4 video standard," IEEE Trans. Circuits and Systems for Video Technology, vol. 11, no. 3, pp. 301-317, 2001.

[17] M. van der Schaar and H. Radha, "A hybrid temporal-SNR fine-granular scalability for Internet video," IEEE Trans. Circuits and Systems for Video Technology, vol. 11, no. 3, pp. 318$331,2001$.

[18] F. Wu, S. Li, and Y.-Q. Zhang, "DCT-prediction based progressive fine granularity Scalability coding," in Proc. International Conference on Image Processing (ICIP '00), vol. 3, pp. 566-569, Vancouver, British Columbia, Canada, September 2000.

[19] F. Wu, S. Li, and Y.-Q. Zhang, "A framework for efficient progressive fine granularity scalable video coding," IEEE Trans. Circuits and Systems for Video Technology, vol. 11, no. 3, pp. 332-344, 2001.
[20] X. Sun, F. Wu, S. Li, W. Gao, and Y.-Q. Zhang, "Macroblockbased progressive fine granularity scalable video coding," in Proc. IEEE International Conference on Multimedia and Expo (ICME '01), pp. 461-464, Tokyo, Japan, August 2001.

[21] F. Wu, S. Li, B. Zeng, and Y.-Q. Zhang, "Drifting reduction in progressive fine granular scalable video coding," in Proc. Picture Coding Symposium, Seoul, Korea, April 2001.

[22] X. Sun, F. Wu, S. Li, W. Gao, and Y.-Q. Zhang, "Macroblockbased progressive fine granularity scalable (PFGS) video coding with flexible temporal-SNR scalablilities," in Proc. International Conference on Image Processing, pp. 1025-1028, Thessaloniki, Greece, October 2001.

[23] Q. Wang, F. Wu, S. Li, Y. Zhong, and Y.-Q. Zhang, "Finegranularity spatially scalable video coding," in Proc. IEEE Int. Conf. Acoustics, Speech, Signal Processing, pp. 1801-1804, Salt Lake City, Utah, USA, May 2001.

[24] R. Yan, F. Wu, S. Li, R. Tao, and Y. Wang, "Efficient video coding with hybrid spatial and fine-grain SNR scalabilities," in Proc. SPIE: Visual Communications and Image Processing 2002, vol. 4671, pp. 850-859, San Jose, Calif, USA, January 2002.

[25] R. Kalluri and M. van der Schaar, Single-loop motioncompensated based fine-granular scalability (MC-FGS) with cross-checked results, ISO/IEC JTC1/SC29/WG11, M6831, Pisa, Italy, 2001.

[26] A. Reibman, L. Bottou, and A. Basso, "DCT-based scalable video coding with drift," in Proc. International Conference on Image Processing, pp. 989-992, Thessaloniki, Greece, October 2001.

[27] A. Reibman and L. Bottou, "Managing drift in DCT-based scalable video coding," in Proc. IEEE Data Compression Conference, pp. 351-360, Salt Lake City, Utah, USA, April 2001.

[28] W.-H. Peng and Y. K. Chen, "Mode-adaptive fine granularity scalability," in Proc. International Conference on Image Processing, pp. 993-996, Thessaloniki, Greece, October 2001.

[29] D. Wu, Y. T. Hou, W. Zhu, et al., "On end-to-end architecture for transporting MPEG-4 video over the Internet," IEEE Trans. Circuits and Systems for Video Technology, vol. 10, no. 6, pp. 923-941, 2000.

[30] S. Floyd and K. Fall, "Promoting the use of end-to-end congestion control in the Internet," IEEE/ACM Transactions on Networking, vol. 7, no. 4, pp. 458-472, 1999.

[31] M. Handley, S. Floyd, J. Padhye, and J. Widmer, TCP friendly rate control (TFRC): Protocol specification, Internet Engineering Task Force, Internet draft, RFC 3448, January 2003.

[32] A. E. Mohr, E. A. Riskin, and R. E. Ladner, "Unequal loss protection: graceful degradation of image quality over packet erasure channels through forward error correction," IEEE Journal on Selected Areas in Communications, vol. 18, no. 6, pp. 819-828, 2000.

[33] P. A. Chou and Z. Miao, "Rate-distortion optimized senderdriven streaming over best-effort networks," in Proc. IEEE 4th Workshop on Multimedia Signal Processing, pp. 587-592, Cannes, France, October 2001.

[34] Video group, Information technology-Coding of audio-visual objects part 5, Amendment 1: Reference software for MPEG-4, ISO/IEC JTC1/SC29/WG11, MPEG M4711, Jeju, March 2002.

[35] F. Wu, X. Sun, and S. Li, Comparisons between PFGS and JVT SP, ISO/IEC JTC1/SC29/WG11, MPEG m8426, Fairfax, 2002.

[36] Test group, MPEG-4 visual fine granularity scalability tools verification test plan, ISO/IEC JTC1/SC29/WG11, MPEG N4456, Pattaya, Thailand, 2001.

[37] Test group, Report on MPEG-4 visual fine granularity scalability tools verification test, ISO/IEC JTC1/SC29/WG11, MPEG N4791, Fairfax, 2002. 
Feng Wu received his B.S. degree in electrical engineering from the University of Xi' an Electrical Science and Technology, Xi'an, China, in 1992, and his M.S. and Ph.D. degrees in computer science from Harbin Institute of Technology, Harbin, China, in 1996 and 1999, respectively. He joined Microsoft Research Asia, Beijing, China, as an Associate Researcher in 1999 and was promoted to a Researcher in 2001. He has

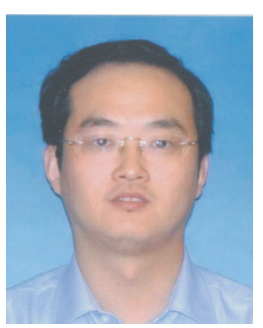
played a major role in Internet Media Group in developing scalable video coding and streaming technologies. He has authored and coauthored over 60 papers in video compression and contributed some technologies to MPEG-4 and H.264. His research interests include video and audio compression, multimedia transmission, and video segmentation.

Honghui Sun received his B.S. degree from Zhejiang University, Hang Zhou, China, in 1992, and his M.S. degree in computer graphics from Beijing University, Beijing, China, in 1995, all in computer science. He was a Lecturer in Computer Science Department, Beijing University, Beijing, China, from 1995 to 1999 . He joined Microsoft Research Asia, Beijing, China as a Research Software Design Engineer in 1999 and was

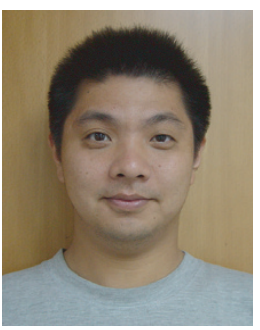
promoted to Senior Research Software Design Engineer in 2001. His work mainly focuses on video compression, multimedia transmission, and network technology.

Guobin Shen received his B.S. degree from Harbin University of Engineering, Harbin, China, in 1994, his M.S. degree from Southeast University, Nanjing, China, in 1997, and his Ph.D. degree from the Hong Kong University of Science and Technology (HKUST) in 2001, all in electrical engineering. He was a Research Assistant at HKUST from 1997 to 2001. Since then, he has been with Microsoft Research Asia. His

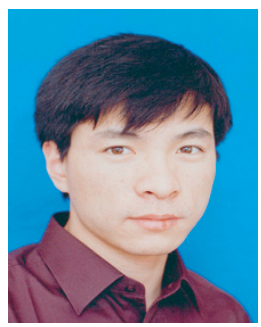
research interests include digital image and video signal processing, video coding and streaming, peer-to-peer networking, and parallel computing.

Shipeng Li received his B.S. and M.S. degrees from the University of Science and Technology of China (USTC) in 1988 and 1991, respectively, and the Ph.D. degree from Lehigh University, Bethlehem, PA, in 1996, all in electrical engineering. He was with the Electrical Engineering Department, University of Science and Technology of China, Hefei, China, from 1991 to 1992. He was a member of the technical

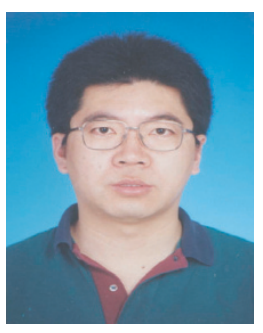
staff at Sarnoff Corporation, Princeton, NJ, from 1996 to 1999. He has been a Researcher with Microsoft Research China, Beijing, since May 1999. His research interests include image/video compression and communications, digital television, multimedia, and wireless communication. He has contributed some technologies to MPEG-4 and H.264.
Ya-Qin Zhang received the B.S. and M.S. degrees in electrical engineering from the University of Science and Technology of China (USTC), Hefei, Anhui, China, in 1983 and 1985, and the Ph.D. degree in electrical engineering from George Washington University, Washington, DC, in 1989. $\mathrm{He}$ is currently the Managing Director of Microsoft Research Asia, Beijing, China, in 1999. He has authored and coauthored over

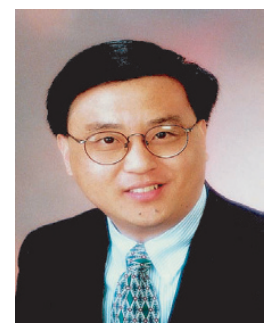
200 refereed papers in leading international conferences and journals, and has been granted over 40 US patents in digital video, Internet, multimedia, wireless, and satellite communications. Dr. Zhang served as Editor-in-Chief for the IEEE Trans. on Circuits and Systems for Video Technology from July 1997 to July 1999. He was the Chairman of the Visual Signal Processing and Communications Technical Committee of the IEEE Circuits and Systems (CAS) Society. He has received numerous awards, including several industry technical achievement awards and IEEE awards, such as the CAS Jubilee Golden Medal. He recently received the Outstanding Young Electrical Engineer of 1998 Award.

Bruce Lin received his B.S. degree from National Taiwan University in 1988 and his M.S. and Ph.D. degrees from the University of Maryland, College Park, in 1994 and 1996, respectively, all in computer science. He was a Research Assistant at the center for automatic research at the University of Maryland from 1992 to 1995 . Since 1995, he has been working with Microsoft on video compression. Currently, he is a Develop-

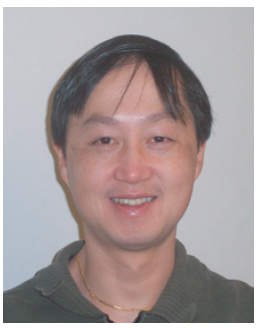
ment Manager of Media Processing Technology group in Microsoft Digital Media Division. His focus is on Windows media video and various image/video processing components for Windows.

Ming-Chieh Lee was born in Taiwan. He received his B.S. degree in electrical engineering from the National Taiwan University, Taiwan, in 1988, and his M.S. and Ph.D. degrees in electrical engineering from California Institute of Technology, Pasadena, in 1991 and 1993, respectively. His Ph.D. research topic was on still and moving image compression using multiscale techniques. From January 1993 to December 1993, he

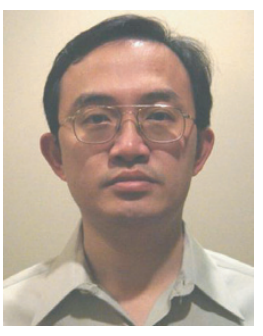
was with the Jet Propulsion Laboratory as a member of the technical staff and was working on multiresolution image transmission and enhancement. In December 1993, he joined the advanced video compression group of Microsoft Corporation, Redmond, Wash, as a Software Design Engineer. He is now the Product Unit Manager in charge of the Core Media Processing Technology group in Microsoft Digital Media Division. His group has produced technologies including Windows media video, Windows media audio, Windows media audio Professional, and Windows media audio voice. 